\title{
ON THE RANGE OF $\mathbf{R}^{2}$ OR $\mathbf{R}^{3}$-VALUED HARMONIC MORPHISMS
}

\author{
BY F. DUHEILLE \\ Université de Lyon 1
}

\begin{abstract}
We prove that, under some general assumptions, the range of any nonconstant harmonic morphism from a simply connected open set $U$ in $\mathbf{R}^{\mathrm{n}}$ to $\mathbf{R}^{3}, \mathrm{n}>3$, cannot avoid three concurrent half-lines, which is an extension to Picard's little theorem. To this end, we will prove two results concerning the windings of Brownian motion around three concurrent half-lines in $\mathbf{R}^{3}$ and the recurrence of some domains linked with the harmonic morphism.
\end{abstract}

1. Introduction. Harmonic morphisms between Euclidean spaces (and more generally between Riemannian manifolds) have been introduced by Fuglede [7] in 1978, and some special cases have been studied by Baird and Wood (see, e.g., [1]) and Gudmundsson [8]. Bernard, Campbell and Davie [2] have considered the probabilistic point of view. Let us recall the definition of such objects.

Definition 1.1. A continuous map $\mathrm{f}: \mathrm{U} \rightarrow \mathbf{R}^{\mathrm{p}}$, defined on a domain $U$ of $\mathbf{R}^{\mathrm{n}}$, is called a harmonic morphism if, for any open set $V$ in $\mathbf{R}^{\mathrm{p}}$ such that $\mathrm{f}^{-1}(\mathrm{~V}) \neq \varnothing$ and for any harmonic function $\mathrm{h}$ on $\mathrm{V}$, the composite function $\mathrm{h} \circ \mathrm{f}: \mathrm{f}^{-1}(\mathrm{~V}) \rightarrow \mathbf{R}$ is harmonic.

The following proposition characterizes harmonic morphisms.

Proposition 1.2. Consider an open set $U \subset \mathbf{R}^{\mathrm{n}}$ and a map in the class $\mathrm{C}^{2}, \mathrm{f}: \mathrm{U} \rightarrow \mathbf{R}^{\mathrm{p}}$. The function $\mathrm{f}$ is a harmonic morphism if and only if each coordinate $f_{i}$ is harmonic and if their gradients are orthogonal and have the same norm:

$$
\left\langle\nabla \mathrm{f}_{\mathrm{i}}, \nabla \mathrm{f}_{\mathrm{j}}\right\rangle=\lambda^{2}(\mathrm{x}) \delta_{\mathrm{ij}}
$$

This proposition leads us to a precise description of harmonic morphisms based on the dimensions of the considered E uclidean spaces. I ndeed, Fuglede [7] and Baird and Wood [1] have shown the following.

TheOREM 1.3. (i) The harmonic morphisms from $\mathbf{R}^{\mathrm{n}}$ to $\mathbf{R}$ are the harmonic functions on $\mathbf{R}^{\mathrm{n}}$ [7].

Received December 1995; revised April 1997.

AMS 1991 subject classifications. 58E20, 31C05, 60J 65, 60J 45.

Key words and phrases. Harmonic morphism, Picard's theorem, Brownian motion, probabilistic potential theory. 
(ii) If $\mathrm{n}<\mathrm{p}$, any harmonic morphism from a domain $\mathrm{U} \subset \mathbf{R}^{\mathrm{n}}$ to $\mathbf{R}^{\mathrm{p}}$ is constant [7].

(iii) If $\mathrm{n}>2$, any nonconstant harmonic morphism from a domain $\mathrm{U} \subset \mathbf{R}^{\mathrm{n}}$ to $\mathbf{R}^{\mathrm{n}}$ is a constant times an affineorthogonal map on $\mathbf{R}^{\mathrm{n}}$ [7].

(iv) The harmonic morphisms from $U \subset \mathbf{R}^{2} \simeq \mathbf{C}$ to $\mathbf{C}$ are the holomorphic or antiholomorphic functions on $\mathrm{U}$. More generally, complex-val ued hol omorphic functions of $n$ complex variables are harmonic morphisms on $\mathbf{C}^{n}$.

(v) Any nonconstant harmonic morphism from $\mathbf{R}^{3}$ to $\mathbf{R}^{2}$ is an orthogonal projection from $\mathbf{R}^{3}$ on a two-dimensional subspace, composed by a holomorphic or antiholomorphic function on $\mathbf{R}^{2}[1]$.

Harmonic morphisms admit a probabilistic interpretation. It is, in fact, a generalization of a classical result of Lévy [11] that claims that plane Brownian motion is invariant under any conformal transformation. More precisely, Bernard, Campbell and Davie [2] have proved the following.

TheOREM 1.4. Let $\mathrm{f}: \mathrm{U} \rightarrow \mathbf{R}^{\mathrm{p}}$ be a continuous map defined on a domain $\mathrm{U}$ of $\mathbf{R}^{\mathrm{n}}$ and let $\left(\mathrm{B}_{\mathrm{t}}\right)_{\mathrm{t} \geq 0}$ be brownian motion on $\mathbf{R}^{\mathrm{n}}$, issued from $\mathrm{B}_{0} \equiv \mathrm{b}_{0} \in \mathrm{U}$. Note $\tau=\inf \left\{\mathrm{t}: \mathrm{B}_{\mathrm{t}} \notin \mathrm{U}\right\}$ and $\rho(\mathrm{t})=\int_{0}^{\mathrm{t}}\left\|\nabla \mathrm{f}\left(\mathrm{B}_{\mathrm{s}}\right)\right\|^{2} \mathrm{ds}$, for all $\mathrm{t}<\tau$.

The map $f$ is a harmonic morphism if and only if the paths of the process $\left(f\left(B_{t}\right)\right)_{0 \leq t \leq \tau}$ are Brownian paths on $\mathbf{R}^{p}$, that is, there exists a Brownian motion $\left(\bar{B}_{\mathrm{s}}\right)_{\mathrm{s} \geq 0}$ on $\mathbf{R}^{\mathrm{p}}$, issued from $\overline{\mathrm{B}}_{0} \equiv \mathrm{f}\left(\mathrm{b}_{0}\right)$, such that $\mathrm{f}\left(\mathrm{B}_{\mathrm{t}}\right)=\overline{\mathrm{B}}(\rho(\mathrm{t}))$.

In such a context, it is natural to search for information on the range $f(U)$ of a harmonic morphism $\mathrm{f}: \mathrm{U} \rightarrow \mathbf{R}^{\mathrm{p}}$, where $\mathrm{U}$ is a domain in $\mathbf{R}^{\mathrm{n}}$. In this article, we prove that, under some general assumptions on $f$ and $U$, the range of any nonconstant $\mathbf{R}^{3}$-valued harmonic morphism $f$ can not avoid three concurrent half-lines, which is of course an extension to Picard's little theorem, according to which the range of any nonconstant entire function on C avoids at most one point. Our result has already been announced in [4]. The proof we give of this fact is based on the invariance property of Theorem 1.4 , and has been inspired by the probabilistic proof of Picard's little theorem due to [3] (see also [5]). However, transience of Brownian motion in $\mathbf{R}^{\mathrm{n}}$, $\mathrm{n} \geq 3$, implies new technical difficulties.

2. Notations and main result. Let us give a few notations: let $h$ : $\mathrm{U} \rightarrow \mathbf{R}$ be a harmonic function on a domain $\mathrm{U}$ of $\mathbf{R}^{\mathrm{n}}$. For any unbounded open set $\mathrm{V} \subset \mathrm{U}$, we note

and

$$
M(r, V, h)=\sup \{|h(x)| ;\|x\| \leq r \text { and } x \in V\}
$$

$$
I(V, h)=\liminf _{r \rightarrow \infty} \frac{\ln M(r, V, h)}{\ln r}
$$

Let us recall the definition of a recurrent set [13]. 
DefinItion 2.1. A Borelian set $\mathrm{C}$ in $\mathbf{R}^{\mathrm{n}}$ is recurrent for the Brownian motion $\left(B_{t}\right)_{t \geq 0}$ in $\mathbf{R}^{n}$ if and only if, $\omega$-almost surely, for all $A>0$, there exists $\tau(\omega)>$ A such that $B_{\tau}(\omega) \in C$. Otherwise, the set $C$ is transient.

Definition 2.2. Consider a Borelian set $\mathrm{C}$ in $\mathbf{R}^{\mathrm{n}}$ and a Brownian motion $\left(B_{t}\right)_{t \geq 0}$ on $\mathbf{R}^{n}$ issued from $B_{0} \equiv b_{0} \notin C$. The set $C$ is polar if and only if, $\omega$-almost surely, the path $\left(B_{t}(\omega)\right)_{t \geq 0}$ does not visit $C$.

Our main result is the following.

THEOREM 2.3. Consider a simply connected domain $U$ in $\mathbf{R}^{\mathrm{n}}$ such that $\mathbf{R}^{n} \backslash U$ is polar. Let $f=\left(f_{1}, \ldots, f_{p}\right): U \rightarrow \mathbf{R}^{p}, n>p, p \in\{2,3\}$, be a harmonic morphism, and let $\left(B_{t}\right)_{t \geq 0}$ be a Brownian motion in $\mathbf{R}^{n}$ issued from $\mathrm{B}_{0} \equiv \mathrm{b}_{0} \subset \mathrm{U}$. We suppose that:

( $\alpha$ ) Almost surely, $\int_{0}^{+\infty}\left\|\nabla f_{1}\left(B_{s}\right)\right\|^{2} \mathrm{ds}=+\infty$;

$(\beta)$ The set $f^{-1}(H)$ admits a recurrent connected component for some open half-space $\mathrm{H}$ in $\mathbf{R}^{\mathrm{p}}$.

Then:

(i) If $p=2, f(U)$ avoids at most one point in $\mathbf{R}^{2} \backslash H$.

(ii) If $p=3$, for all $x_{0} \in \mathbf{R}^{3} \backslash H$,

$$
\operatorname{card}\left\{\mathrm{u} \in \mathbf{S}^{2}, \mathrm{x}_{0}+\mathbf{R}^{+} \mathrm{u} \subset \mathbf{R}^{3} \backslash(\mathrm{f}(\mathrm{U}) \cup \mathrm{H})\right\} \leq 2,
$$

where $\mathbf{S}^{2}$ is the unit sphere in $\mathbf{R}^{3}$.

Remark. Assumptions $(\alpha)$ and $(\beta)$ are always checked if $\mathrm{f}$ is a polynomial harmonic morphism.

The hypothesis $(\beta)$ involves only one coordinate function of the harmonic morphism, which is in fact a harmonic function. We give here a condition, on harmonic functions, that implies hypothesis $(\beta)$.

THEOREM 2.4. Let $h$ be a harmonic function on $U$ where $U$ is a domain of $\mathbf{R}^{\mathrm{n}}$ of polar complement. Then any connected component $\mathrm{C}$ of $\mathrm{h}^{-1}\left(\mathbf{R}_{+}^{*}\right)$ such that $\mathrm{I}(\mathrm{C}, \mathrm{h})$ is finite and such that any point in $\partial \mathrm{C}$ is regular for $\mathrm{C}$, is recurrent.

One could think that, for any harmonic function $\mathrm{h}$ on $\mathbf{R}^{\mathrm{n}}$, one at least of the connected components of $h^{-1}\left(\mathbf{R}_{+}^{*}\right)$ or of $h^{-1}\left(\mathbf{R}_{-}^{*}\right)$ is recurrent. The following example shows this is false.

Example. Consider the function $\mathrm{h}: \mathbf{R}^{4} \rightarrow \mathbf{R}$ defined by

$$
h(x, y, z, t)=\cos x \cos y \cos z e^{\sqrt{3} t} \text {. }
$$

This function is clearly harmonic on $\mathbf{R}^{4}$, but no connected component of $\mathrm{h}^{-1}\left(\mathbf{R}_{+}^{*}\right)$ or of $\mathrm{h}^{-1}\left(\mathbf{R}_{-}^{*}\right)$ is recurrent. 
3. Proof of Theorem 2.3. As our method can also be used to solve the case $p=2$, we will study more carefully the case $p=3$. This theorem is based on two essential facts. We will prove that Brownian paths in $\mathbf{R}^{3}$ get more and more tangled in their windings around three concurrent half-lines. An argument based on homotopy allows us to conclude.

Notation. Later on in this article, we will note $E=\mathbf{R}^{3} \backslash U_{i=1}^{3} D_{i}$, where $D_{1}, D_{2}, D_{3}$ are three concurrent half-lines.

DEFINITION 3.1. Let us note $T$ the border of the infinite trihedral defined by the three concurrent half-lines $D_{i}, i=1,2,3$, and consider a curve $\Gamma:[0, \mathrm{t}] \rightarrow \mathrm{E}$.

(i) We will say that $\Gamma_{[0, t]}$ is unwound in $E$ (around the three half-lines) if there exists a curve $\tilde{\Gamma}:[0,1] \rightarrow \mathrm{E}$ such that:

(a) $\tilde{\Gamma}(0)=\Gamma(\mathrm{t}), \tilde{\Gamma}(1)=\Gamma(0)$

(b) $\operatorname{card}(\tilde{\Gamma}[0,1] \cap \mathrm{T}) \leq 2$;

(c) $\Gamma_{[0, t]} * \Gamma_{[0,1]}$ is homotopic to a point in $\mathrm{E}$.

Otherwise, we will say $\Gamma$ is wound in $E$.

(ii) We will say that a curve $\Gamma:[0,+\infty) \rightarrow E$ comes wound if, for all t big enough, the curve $\Gamma_{[0, t]}$ is wound.

Then we can state the following result.

THEOREM 3.2. Let $\left(\bar{B}_{t}\right)_{t \geq 0}$ be an $\mathbf{R}^{3}$-valued Brownian motion issued from a point $\bar{B}_{0} \equiv b_{0} \in E$. Then, $\omega$-almost surely, the path $\left(\bar{B}_{t}(\omega)\right)_{t \geq 0}$ comes wound around the three concurrent half-lines $D_{1}, D_{2}, D_{3}$.

RemARK. As straight lines are polar sets for three-dimensional Brownian motion, the winding of Brownian paths around three half-lines is well defined.

Let us admit for a while Theorem 3.2 and deduce from it Theorem 2.3.

Assume that $f(U)$ avoids three concurrent half-lines $D_{1}, D_{2}, D_{3}$ included in the half-space $\mathrm{H}^{-}(\mathrm{a}, \mathrm{b})$ and that a connected component $\mathrm{C}$ of the open set $\mathrm{f}^{-1}\left(\mathrm{H}^{-}(\mathrm{a}, \mathrm{b})\right)$ is recurrent. We will build, using Brownian motion on $\mathbf{R}^{\mathrm{n}}, \mathrm{a}$ closed curve $\Gamma$ in $U$ whose image by $f: U \rightarrow E=\mathbf{R}^{3} \backslash U_{i=1}^{3} D_{i}$ is not homotopic to a point in $E$ (thanks to Theorem 3.2), which is absurd. Indeed, as we have supposed $U$ simply connected, the closed curve $\Gamma$ is homotopic to a point and its image under the continuous map $f$ should be homotopic to a point in $f(U)$.

Let $\left(B_{t}\right)_{t \geq 0}$ be a Brownian motion on $\mathbf{R}^{n}$. We will suppose $B_{0} \equiv b_{0} \in C$ and note:

$$
\Omega_{0}=\left\{\omega \in \Omega,\left(B_{t}\right)_{t \geq 0} \text { does not visit } \mathbf{R}^{\mathrm{n}} \backslash \mathrm{U}\right\} .
$$

As $\mathbf{R}^{\mathrm{n}} \backslash \mathrm{U}$ is polar, $\mathrm{P}\left(\Omega_{0}\right)=1$. 
By Theorem 3.2, the paths of $\left(f\left(B_{t}\right)\right)_{t \geq 0}$ come wound almost surely around the three half-lines, because the paths of this process are Brownian paths in $\mathbf{R}^{3}$, and the assumption $(\alpha)$ implies we find whole Brownian paths. Let us note:

$$
\Omega_{1}=\left\{\omega \in \Omega, \text { there exists } \mathrm{S}_{\omega} \geq 0, \mathrm{f}\left(\mathrm{B}_{[0, \mathrm{t}]}\right) \text { is wound for all } \mathrm{t} \geq \mathrm{S}_{\omega}\right\} .
$$

We have then $\mathrm{P}\left(\Omega_{1}\right)=1$.

Set

$$
\Omega_{2}=\left\{\omega \in \Omega, \forall \mathrm{A}>0, \exists \tau(\omega, \mathrm{A})>\mathrm{A}, \mathrm{B}_{\tau} \in \mathrm{C}\right\} .
$$

As we supposed $C$ recurrent, we have $P\left(\Omega_{2}\right)=1$, and $P\left(\Omega_{0} \cap \Omega_{1} \cap \Omega_{2}\right)=1$.

Fix then $\omega \in \Omega_{0} \cap \Omega_{1} \cap \Omega_{2}$. For such an $\omega$, there exists $\sigma=\sigma(\omega) \in \mathbf{R}^{+}$ such that for all $\mathrm{t} \geq \sigma(\omega)$, the path $\left(\mathrm{f}\left(\mathrm{B}_{\mathrm{s}}(\omega)\right)\right)_{\mathrm{s} \in[0, \mathrm{t}]}$ is wound in $\mathrm{E}$. We can then choose $\tau(\omega) \geq \sigma(\omega)$ such that $\mathrm{B}_{\tau}(\omega)$ is in C.

The set $C$ is connected and open: it is then arcwise-connected, and there exists a curve $\gamma$ in $C$ linking $B_{\tau}(\omega)$ and $B_{0}(\omega)$. The curve $f(\gamma)$ closes the path $f\left(B_{[0, \tau]}\right)$ according to the rules of Definition 3.1. By definition of $\tau$, the closed curve $\mathrm{f}\left(\mathrm{B}_{[0, \tau]} * \gamma\right)$ can not be homotopic to a point in $\mathrm{f}(\mathrm{U}) \subset \mathrm{E}$. The curve $\mathrm{B}_{[0, \tau]} * \gamma$ forms the announced curve.

4. Proof of Theorem 3.2. Let $D_{1}, D_{2}, D_{3}$ be three concurrent half-lines in $\mathbf{R}^{3}$, issued from 0 . We note $D_{i}=\mathbf{R}^{+} u_{i}$ with $\left\|u_{i}\right\|=1, i=1,2$, 3. Consider the map

$$
\begin{gathered}
\pi: \mathbf{R}^{3} \backslash \mathrm{D}_{1} \rightarrow \mathbf{R}^{2} \simeq \mathrm{D}_{1}^{\perp} \\
\mathrm{x} \rightarrow \pi(\mathrm{x})=\frac{\mathrm{x}-\left\langle\mathrm{x}, \mathrm{u}_{1}\right\rangle \mathrm{u}_{1}}{\|\mathrm{x}\|-\left\langle\mathrm{x}, \mathrm{u}_{1}\right\rangle}
\end{gathered}
$$

Lemma 4.1. The map $\pi$ is a harmonic morphism from $\mathbf{R}^{3} \backslash D_{1}$ to $\mathbf{R}^{2}$.

Indeed, $\pi$ is the composite map of $\phi: x \rightarrow \mathrm{x} /\|\mathrm{x}\|$ and of the stereographic projection of pole $u_{1}$ onto the plane orthogonal to $D_{1}$ through 0 . The image by $\phi$ of an $\mathbf{R}^{3}$-valued Brownian motion is a time change of a spherical Brownian motion and the image by the stereographic projection of a spherical Brownian motion is a time change of a plane Brownian motion: the paths of the image by $\pi$ of an $\mathbf{R}^{3}$-valued Brownian motion are plane Brownian paths. Theorem 1.4 allows us to claim that $\pi$ is a harmonic morphism.

Lemma 4.2. We have $\pi\left(\mathbf{R}^{3} \backslash \mathrm{U}_{\mathrm{i}=1}^{3} \mathrm{D}_{\mathrm{i}}\right)=\mathbf{R}^{2} \backslash\left\{\pi\left(\mathrm{u}_{2}\right), \pi\left(\mathrm{u}_{3}\right)\right\}$.

One can easily check that the inverse image by $\pi$ of any point in $\mathbf{R}^{2}$ is a half-line issued from 0 , and distinct from $D_{1}$, which proves this lemma.

We can now prove Theorem 3.2. To that effect, consider a Brownian motion $\left(\bar{B}_{t}\right)_{t \geq 0}$ in $\mathbf{R}^{3}$ issued from $\bar{B}_{0} \equiv \bar{b}_{0} \notin U_{i=1}^{3} D_{i}$. The process $\left.\left(\pi\left(\bar{B}_{t}\right)\right)_{t \geq 0}\right)$ is a time change of a Brownian motion on the plane minus $\left\{\pi\left(u_{2}\right), \pi\left(u_{3}\right)\right\}$, and the change of time converges almost surely to $+\infty$ as $t \rightarrow+\infty$. We will now use a 
result of McKean [12] (see also [3] and [5]) according to which plane Brownian motion comes wound around two different points.

LEMmA 4.3. Let $\left(B_{t}\right)_{t \geq 0}$ be a plane Brownian motion issued from $B_{0} \equiv b_{0}$ and $x_{1}, x_{2}$, two distinct points in $\mathbf{R}^{2}$, different from $b_{0}$. Then, $\omega$-almost surely, there exists $T(\omega)>0$ such that for all $t>T(\omega)$, the path $\left(B_{s}(\omega)\right)_{0 \leq s \leq t}$ is wound in $\mathbf{R}^{2} \backslash\left\{\mathrm{x}_{1}, \mathrm{x}_{2}\right\}$.

REMARK. One can easily define the winding of any unbounded curve in $\mathbf{R}^{2}$ minus two points as one did in $\mathbf{R}^{3}$ minus three half-lines.

Let $\mathrm{T}$ be a time such that $\left(\pi\left(\bar{B}_{s}\right)\right)_{0 \leq s \leq t}$ is wound in $\mathbf{R}^{2} \backslash\left\{\pi\left(\mathrm{u}_{2}\right), \pi\left(\mathrm{u}_{3}\right)\right\}$ for all $t>T$, and choose any curve $\Gamma$ in $\mathbf{R}^{3} \backslash U_{i=1}^{3} D_{i}$ that closes the path $\left.\left(\bar{B}_{s}\right)\right)_{0 \leq s \leq t}$ according to the rules of Definition 3.1. As the curve $\pi(\Gamma)$ closes $\pi\left(\bar{B}_{[0, t]}\right)$, the plane closed curve $\pi\left(\bar{B}_{[0, t]} * \Gamma\right)$ can not be homotopic to a point in $\mathbf{R}^{2} \backslash\left\{\pi\left(u_{2}\right), \pi\left(u_{3}\right)\right\}$. Hence, the closed curve $\bar{B}_{[0, t]} * \Gamma$ is not homotopic to a point in $\mathbf{R}^{3} \backslash \cup_{i=1}^{3} D_{i}$, which proves that $\mathbf{R}^{3}$-valued Brownian motion comes wound around three concurrent half-lines in $\mathbf{R}^{3}$.

5. Proof of Theorem 2.4. Let us first prove the following lemma.

LEMmA 5.1. Let $h: U \rightarrow \mathbf{R}$ be a harmonic function on an open connected set of polar complement and consider a connected component $\mathrm{C}$ of $\mathrm{h}^{-1}\left(\mathbf{R}_{+}^{*}\right)$.

If $\partial \mathrm{C}$ is regular for $\mathrm{C}$ and if $\mathrm{I}(\mathrm{C}, \mathrm{h})$ is finite, the harmonic function $\mathrm{h}$ admits a continuous extension on the set $\bar{C}$.

Proof of Lemma 5.1. At first, choose a point $x_{0}$ in $\stackrel{\circ}{\bar{C}} \backslash \mathrm{C}$. As $I(C, h)$ is finite and $\stackrel{\circ}{\bar{C}} \backslash C$ is polar, the harmonic function $h$ is bounded in a vicinity of $x_{0}$ in $U$. Hence, it admits a limit at that point (see, e.g., [10], page 271]). We can then suppose $\mathrm{C}=\overline{\mathrm{C}}$.

Consider now, for any $r>0$, the bounded harmonic function $\tilde{h}_{r}$ on $C \cap B_{r}$ (where $B_{r}$ is the ball of center 0 and radius $r$ ) such that

$$
\begin{array}{ll}
\tilde{h}_{r}=h & \text { on } S_{r} \cap C, \\
\tilde{h}_{r} \equiv 0 & \text { on } \partial C \cap B .
\end{array}
$$

As $\partial \mathrm{C}$ is regular for $\mathrm{C}, \mathrm{h}_{\mathrm{r}}$ does exist and is unique because $C_{r}$ is bounded.

We have then $\tilde{h}_{r}=h$ on $\partial \mathrm{C}$, except maybe on a polar set of $\overline{\mathrm{C}}$. By unicity of the solution of the Dirichlet problem on $C \cap B_{r}, h=\tilde{h}_{r}$ on $\bar{C} \cap B_{r}$ : the harmonic function $h$ is continuous on $\bar{C}$ and we have $h(x)=0$ for any $\mathrm{x} \in \partial \mathrm{C}$.

Proof of Theorem 2.4. There is no unicity for the Dirichlet problem for the Laplacian on the domain $\mathrm{C}$ : the harmonic function $\mathrm{h}$ is nonconstant on $\mathrm{C}$ and null on its border. Furthermore, we suppose that $h$ does not grow too fast; the set $C$ has to be big enough, and we will show that it is recurrent. 
Let us suppose that $C$ is transient and show that, necessarily, $I(C, h)$ is infinite.

We will use the following lemma, proved by Huber [9] that minorizes the growth of a harmonic function $h$ on a connected component of $h^{-1}\left(\mathbf{R}_{+}^{*}\right)$ :

Lemma 5.2. Let $U \subset \mathbf{R}^{\mathrm{n}}, \mathrm{n} \geq 2$, be an open connected set, $\Gamma=\partial \mathrm{U}$ its border and h: $\mathrm{U} \rightarrow \mathbf{R}$, a subharmonic function such that

$$
\forall x \in \Gamma, \limsup _{y \rightarrow x, y \in U} h(y) \leq 0 \text {. }
$$

Then, one of the two foll owing properties is checked:

(i) We have $\mathrm{h} \leq 0$ on $\mathrm{U}$.

(ii) There exist two constants $K>0$ and $r_{0}>0$ such that, for all $r \geq r_{0}$,

$$
\left(\sup _{x \in U,\|x\|=r} h(x)\right)^{2} \geq K r^{2-n} \int_{r_{0}}^{r} \rho^{n-3} \exp \left(2 \int_{r_{0}}^{\rho} \alpha(s) d s / s\right) d \rho,
$$

where $\alpha(\mathrm{s})$ is the positive root of the second-degree equation $\alpha(\alpha+\mathrm{n}-2)=$ $\lambda_{1}(\mathrm{~s}), \lambda_{1}(\mathrm{~s})$ being the first eigenvalue of the spherical Laplacian on the unit sphere $\mathbf{S}$ in $\mathbf{R}^{\mathrm{n}}$, for the Dirichlet problem on $\mathbf{S} \cap \mathrm{S}^{-1} \mathrm{U}$.

Let us apply Lemma 5.2 to the harmonic function $\mathrm{h}$ and the connected open set $\mathrm{C}$. We have clearly

$$
h(x)=0 \text { for all } x \in \partial C
$$

and

$$
h(x)>0 \text { for all } x \in C .
$$

Here, inequality (1) becomes

$$
M^{2}(r, C, h) \geq K r^{2-n} \int_{r_{0}}^{r} \rho^{n-3} \exp \left(2 \int_{r_{0}}^{\rho} \alpha(s) d s / s\right) d \rho
$$

for all $r \geq r_{0}, K$ and $r_{0}$ being two positive constants.

For all $r \geq 2 r_{0}$, we deduce

$$
\begin{aligned}
M^{2}(r, C, h) \geq K r^{2-n}[ & \int_{r_{0}}^{r / 2} \rho^{n-3} \exp \left(2 \int_{r_{0}}^{\rho} \alpha(s) d s / s\right) d \rho \\
& \left.+\int_{r / 2}^{r} \rho^{n-3} \exp \left(2 \int_{r_{0}}^{r / 2} \alpha(s) d s / s\right) d \rho\right] .
\end{aligned}
$$

This inequality implies

$$
M^{2}(r, C, h) \geq \frac{K}{n-2}\left(1-2^{2-n}\right) \exp \left(2 \int_{r_{0}}^{r / 2} \alpha(s) \frac{d s}{s}\right) .
$$

Set $K_{1}=\frac{1}{2} \ln \left[K\left(1-2^{2-n}\right) /(n-2)\right]$. 
From the overestimate (2), we infer

$$
\frac{\ln \mathrm{M}(\mathrm{r}, \mathrm{C}, \mathrm{h})}{\ln \mathrm{r}} \geq \frac{\mathrm{K}_{1}}{\ln \mathrm{r}}+\frac{1}{\ln \mathrm{r}} \int_{\ln \mathrm{r}_{0}}^{\ln (\mathrm{r} / 2)} \alpha\left(\mathrm{e}^{\mathrm{u}}\right) \mathrm{du},
$$

As $C$ is transient, the area of $\mathbf{S} \cap \mathrm{s}^{-1} \mathrm{C}$ converges to 0 as $\mathrm{s}$ converges to $+\infty$, so that the first eigenvalue of this set converges to $+\infty$. The quantity $\alpha$ (s) converges to $+\infty$ and finally, $\mathrm{I}(\mathrm{C}, \mathrm{h})$ has to be infinite.

Acknowledgment. We thank the referee for having suggested to us this short proof of Theorem 2.4. A more precise result on the growth of $h$ can be obtained using Wiener's test (see, e.g., [13]) and an isoperimetric inequality on the unit sphere $\mathbf{S}$ proved by Friedland and Hayman [6].

\title{
REFERENCES
}

[1] BAIRD, P. and Wood, J . C. (1988). Bernstein theorems for harmonic morphisms from $\mathbf{R}^{3}$ and $\mathbf{S}^{3}$. Math. Ann. 280 579-603.

[2] Bernard, A., Campbell, E. A. and Davie, A. M. (1979). Brownian motion and generalized analytic and inner functions. Ann. Inst. Fourier 29 207-228.

[3] Davis, B. (1975). Picard's theorem and Brownian motion. Trans. Amer. Math. Soc. 213 353-362.

[4] Duheille, F. (1995). Sur l'image des morphismes harmoniques à valeurs dans $\mathbf{R}^{2}$ ou $\mathbf{R}^{3}$. C.R. Acad. Sci. Paris Sér. I Math. 320 1495-1500.

[5] Durrett, R. (1984). Brownian Motion and Martingales in Analysis. Wadsworth, Monterey, CA.

[6] FriedLAND, S. and HAYMAN, W. K. (1976). Eigenvalue inequalities for the Dirichlet problem on spheres and the growth of subharmonic functions. Comment. Math. Helv. 51 133-161.

[7] Fuglede, B. (1978). Harmonic morphisms between Riemannian manifolds. Ann. Inst. Fourier 28 107-144.

[8] Gugmundsson, S. (1994). Harmonic morphisms from complex projective spaces. Geom. Dedicata 53 155-161.

[9] Huber, A. (1952). Über Wachstumseigenschaften gewisser Klassen von Subharmonischen Funktionen. Comment. Math. Helv. 26 81-116.

[10] KellogG, O. D. (1953). Foundations of Potential Theory. Dover, New York.

[11] LÉVY, P. (1948). Processus Stochastiques et Mouvement Brownien. Gauthier-Villars, Paris.

[12] McKean, H. P. (1969). Stochastic Integrals. Academic Press, New York.

[13] Port, S. C. and Stone, C. J. (1978). Brownian Motion and Classical Potential Theory. Academic Press, New York.

\author{
Laboratoire de Probabilités \\ UNIVERSITÉ CLAUDE BERNARD, LYON 1 \\ 43, BOULEVARD DU 11 NOVEMBRE 1918 \\ 69622 Villeurbanne Cedex \\ FRANCE \\ E-MAIL: duheille@ jonas.univ-Iyon1.fr
}

